\title{
Congenital Cerebellar Hypoplasia
}

National Cancer Institute

\section{Source}

National Cancer Institute. Congenital Cerebellar Hypoplasia. NCI Thesaurus. Code C98890.

Hypoplasia of the cerebellum that is associated with inherited metabolic disorders and neurodegenerative disorders. Signs and symptoms include mental and developmental delays, walking and balance difficulties, floppy muscle tone, and seizures. 